harmful than more gradual adjustments. An important caution in interpreting the work of Kiessling et al. is that the researchers measured gene expression at the level of mRNA (16). It is important to determine the dynamics of key proteins and posttranslational modifications of proteins to more fully appreciate the relevance of these findings. Development of methods for noninvasive imaging of circadian clock dynamics in various tissues within an animal would allow studies similar to this one to become more routine and would better equip us to develop effective treatments for jet lag.

Our current understanding of jet lag incorporates disorder at many levels. Disorder can exist between the timing of the organism and the environment, within the different organs in the organism, and among the cells within a single tissue. The study in this issue demonstrates that jet lag may also include disorder among the circadian clock genes within a cell.

\section{Acknowledgments}

Tanya Leise provided helpful feedback to an earlier draft of this commentary.

Address correspondence to: Mary Harrington, Bass Hall 417, Smith College, Psychology Department, Northampton,
Massachusetts 01063, USA. Phone: 413.585.3925; Fax: 413.585.3786; E-mail: mharring@smith.edu.

1. Knutsson A. Health disorders of shift workers. Occup Med (Lond). 2003;53(2):103-108.

2. Megdal SP, Kroenke CH, Laden F, Pukkala E, Schernhammer ES. Night work and breast cancer risk: a systematic review and meta-analysis. Eur J Cancer. 2005;41(13):2023-2032.

3. Mahoney MM. Shift work, jet lag, and female reproduction [published online ahead of print March 8 , 2010]. Int J Endocrinol. doi:10.1155/2010/813764.

4. Filipski E, et al. Effects of chronic jet lag on tumor progression in mice. Cancer Res. 2004. 64(21):7879-7885.

5. Davidson AJ, Sellix MT, Daniel J, Yamazaki S, Menaker M, Block GD. Chronic jet-lag increases mortality in aged mice. Curr Biol. 2006; 16(21):R914-R916.

6. Penev PD, Kolker DE, Zee PC, Turek FW. Chronic circadian desynchronization decreases the survival of animals with cardiomyopathic heart disease. Am J Physiol. 1998;275(6 pt 2):H2334-H2337.

7. Straif K, et al. Carcinogenicity of shift-work, painting, and fire-fighting. Lancet Oncol. 2007;8(12):1065-1066.

8. Dibner C, Schibler U, Albrecht U. The mammalian circadian timing system: organization and coordination of central and peripheral clocks. Annu Rev Physiol. 2010;72:517-549.

9. Ukai H, Ueda HR. Systems biology of mammalian circadian clocks. Annu Rev Physiol. 2010;72:579-603.

10. Yamamoto T, et al. Acute physical stress elevates mouse period 1 mRNA expression in mouse peripheral tissues via a glucocorticoid-responsive element. J Biol Chem. 2005;280(51):42036-42043.

11. Balsalobre A, et al. Resetting of circadian time in peripheral tissues by glucocorticoid signaling. Science. 2000;289(5488):2344-2347.
12. Yamazaki S, et al. Resetting central and peripheral circadian oscillators in transgenic rats. Science. 2000;288(5466):682-685

13. Davidson AJ, Castanon-Cervantes O, Leise TL, Molyneux PC, Harrington ME. Visualizing jet lag in the mouse suprachiasmatic nucleus and peripheral circadian timing system. Eur J Neurosci. 2009;29(1):171-180.

14. Nakamura W, Yamazaki S, Takasu NN, Mishima K, Block GD. Differential response of Period 1 expression within the suprachiasmatic nucleus. J Neurosci. 2005;25(23):5481-487.

15. Nagano $M$, et al. An abrupt shift in the day/night cycle causes desynchrony in the mammalian circadian center. J Neurosci. 2003;23(14):6141-6151.

16. Kiessling S, Eichele G, Oster H. Adrenal glucocorticoids have a key role in circadian resynchronization in a mouse model of jet lag. J Clin Invest. 2010; 120(7):2600-2609.

17. Reddy AB, Field MD, Maywood ES, Hastings MH. Differential resynchronisation of circadian clock gene expression within the suprachiasmatic nuclei of mice subjected to experimental jet lag. J Neurosci. 2002;22(17):7326-7330.

18. Sage $\mathrm{D}$, et al. Influence of the corticosterone rhythm on photic entrainment of locomotor activity in rats. J Biol Rbythms. 2004;19(2):144-156.

19. Agostino PV, Plano SA, Golombek DA. Sildenafil accelerates reentrainment of circadian rhythms after advancing light schedules. Proc Natl Acad Sci US A. 2007;104(23):9834-9839.

20. Kessler EJ, Sprouse J, Harrington ME. NAN-190 potentiates the circadian response to light and speeds re-entrainment to advanced light cycles. Neuroscience. 2008;154(4):1187-1194.

21. Leise T, Siegelmann H. Dynamics of a multistage circadian system. J Biol Rhythms. 2006;21(4):314-323.

22. Dean DA 2nd, Forger DB, Klerman EB. Taking the lag out of jet lag through model-based schedule design. PLoS Comput Biol. 2009;5(6):e1000418.

\title{
An energetic tale of AMPK-independent effects of metformin
}

\author{
Russell A. Miller and Morris J. Birnbaum
}

The Institute for Diabetes, Obesity, and Metabolism, University of Pennsylvania, Philadelphia, Pennsylvania, USA.

\begin{abstract}
Metformin has become a mainstay in the modest therapeutic armamentarium for the treatment of the insulin resistance of type 2 diabetes mellitus. Although metformin functions primarily by reducing hepatic glucose output, the molecular mechanism mediating this effect had remained elusive until recently. Metformin impairs ATP production, activating the conserved sensor of nutritional stress AMP-activated protein kinase (AMPK), thus providing a plausible and generally accepted model for suppression of gluconeogenic gene expression and glucose output. In this issue of the JCI, Foretz et al. refute this hypothesis by showing that AMPK is dispensable for the effects of metformin on hepatic glucose output in primary hepatocytes; rather, their data suggest that the antidiabetic effects of metformin in the liver are mediated directly by reducing energy charge.
\end{abstract}

Conflict of interest: Morris J. Birnbaum is a paid consultant to Pfizer, has stock in Motif Inc., and receives research support from Johnson \& Johnson.

Citation for this article: $J$ Clin Invest. 2010; 120(7):2267-2270. doi:10.1172/JCI43661

\section{Metformin in the treatment of type 2 diabetes}

The increasing worldwide incidence of obesity is driving an epidemic of type 2 diabetes mellitus (T2DM), with global prevalence of the disease predicted to reach $5.4 \%$, or 300 million people by 2025 (1). Individuals are deemed to have T2DM if they have elevated serum glucose levels - a pathology typically preceded by peripheral insulin resistance and hyperinsulinemia without hyperglycemia - manifest by an impaired insulinstimulated glucose uptake and an impaired reduction in glucose output from the liver. $\mathrm{T} 2 \mathrm{DM}$ and insulin resistance are associated with increased incidence of serious cardiovascular morbidity and mortality, and collectively these conditions contribute to the cluster of pathologies termed the metabolic syndrome. A mainstay of pharmacological therapy for individuals with $\mathrm{T} 2 \mathrm{DM}$ is the biguanide drug metformin, one of only two commonly used drugs that 


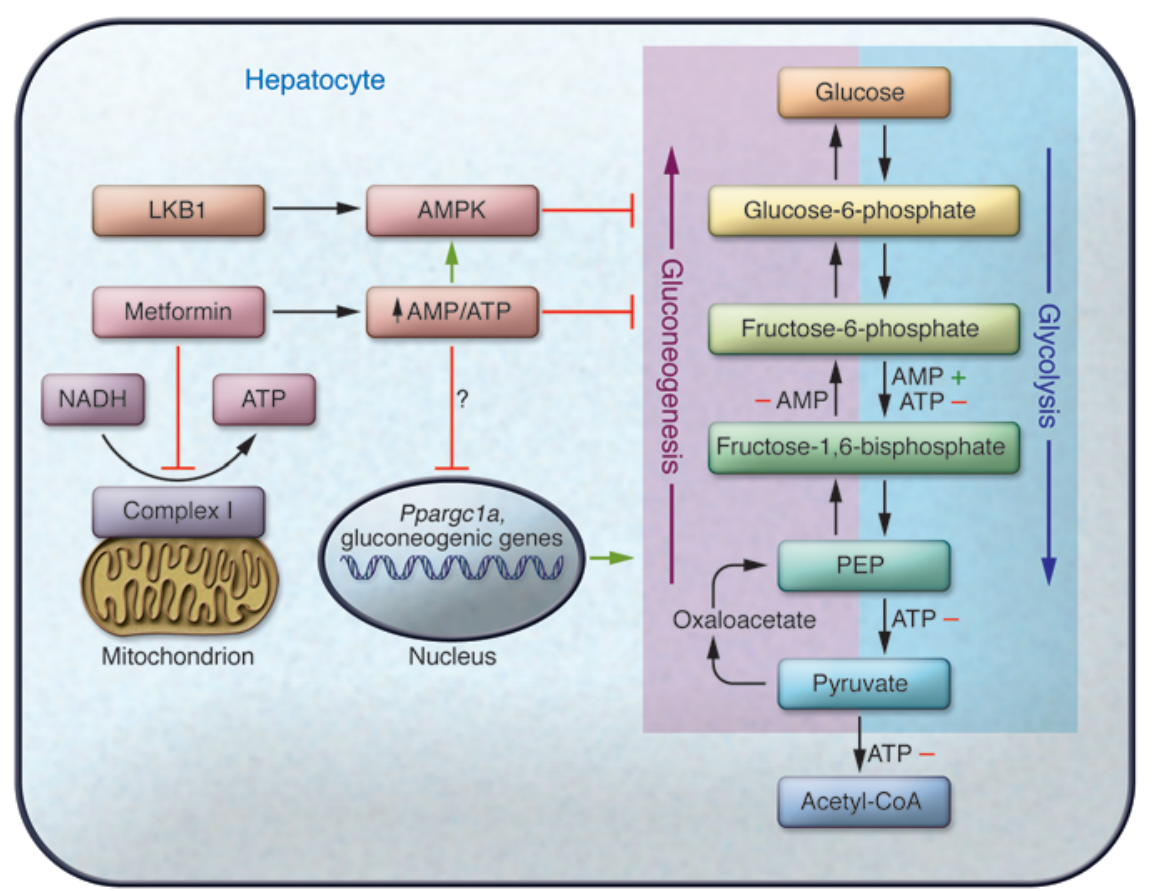

augment or mimic insulin action $(2,3)$. Metformin owes its use as an antidiabetic drug to the recognition in the early 1900 s of the hypoglycemic effects of guanidine, which was subsequently revealed as the active antidiabetic component of the French lilac (Galega officinalis), also known as goat's rue, a traditional botanic medicine used to treat diabetes across medieval Europe $(4,5)$. Metformin and a related biguanide, phenformin, were introduced in the 1950 s to treat T2DM, but phenformin was subsequently removed from the market due to an unacceptable incidence of severe lactic acidosis. Metformin, on the other hand, has proven to be well tolerated and highly efficacious in reducing blood glucose in insulin-resistant individuals, an effect largely attributed to reductions in hepatic glucose output, although some studies have reported increases in peripheral glucose uptake (6). Additional benefits of metformin therapy include improved insulin secretion and decreased cardiovascular risk, although these effects could be secondary to reductions in serum glucose and insulin levels $(7,8)$.

\section{Mechanisms of metformin action}

The primary cellular target for metformin is believed to be complex I of mitochondrial oxidative phosphorylation, although the precise site of interaction has yet to be established (9). Complex I is the sole entry point for reduced NADH to contribute to the maintenance of the mitochondrial proton gradient necessary for ATP production, and its inhibition by metformin and phenformin leads to a reduction in cellular energy charge, a measure of the energetic state of the cells' adenine nucleotide pool defined as $([\mathrm{ATP}]+0.5[\mathrm{ADP}]) /([\mathrm{ATP}]+$ $[\mathrm{ADP}]+[\mathrm{AMP}])$. The metformin-induced reduction in energy charge activates AMPactivated protein kinase (AMPK), which was therefore quite reasonably advanced as the major mediator of the glucose-lowering effects of metformin (10). The ability of a constitutively active mutant of the AMPK catalytic subunit to mimic the actions of metformin further supports this Foretz et al. report studies utilizing genetic ablation of both AMPK catalytic subunits and the upstream activating kinase LKB1 in liver and primary hepatocytes to show clearly that AMPK and LKB1 are dispensable for metformin-induced decreases in hepatic glucose output in mice (12). As expected, metformin and other AMPK activators caused a decrease in cellular ATP and a concomitant increase in AMP, which Foretz et al. suggest are responsible for the metformin-induced reductions in hepatic glucose production.

\section{Energy charge regulation of hepatic glucose output}

Considerable recent research has been devoted to understanding the endocrine idea (11). In this issue of the JCI, however,

\section{Figure 1}

Regulation of hepatic glycolytic/gluconeogenic pathways. Metformin inhibits mitochondrial complex I and increases the AMP/ATP ratio, which leads to the activation of the energysensing kinase AMPK. Foretz et al. (12) show that activation of AMPK is dispensable for metformin-induced reduction in hepatic glucose output. While multiple regulatory points exist for direct AMP- and ATP-mediated effects on glycolysis and gluconeogenesis, the mechanism of energy charge regulation of gluconeogenic gene expression described by Foretz et al. is currently unknown. PEP, phosphoenolpyruvate. control of hepatic glucose output, in particular the opposing actions of the pancreatic hormones insulin and glucagon in maintaining euglycemia. Less well represented in the recent literature are studies of the role of intracellular metabolites as cell-autonomous regulators of hepatic glucose output. The anabolic process of gluconeogenesis is an energetically costly program, requiring 6 ATP equivalents per molecule of glucose synthesized. To maintain normal cellular energy charge, a hepatocyte must balance this energy demand with production, likely through the oxidation of molecules such as fatty acids. In times of poor substrate availability or hypoxia, or upon treatment with metformin, energy charge decreases and glucose output must be reduced accordingly. This energetic expense of glucose synthesis is exemplified in recent studies examining the decreased hepatic energy state associated with prolonged fasting or glucagon treatment in mice (13).

A rich body of literature in the latter half of the twentieth century defined the exquisite control energy charge can exert on gluconeogenic and glycolytic flux via allosteric regulation of key enzymes in this pathway (Figure 1). For example, AMP synergizes with the regulatory sugar fructose 2,6-bisphosphate to stimulate phosphofructokinase and inhibit fructose-1,6bisphosphatase, and a decrease in energy charge activates glycolysis by releasing ATP- 
mediated inhibition of L-type pyruvate kinase and phosphofructokinase (14-16). Additionally, high ATP levels block the entry of pyruvate into the tricarboxylic acid (TCA) cycle by inhibiting pyruvate dehydrogenase (17). These effects alone might explain how the decreased energy charge induced by metformin suppresses hepatic glucose output. In addition to energy charge, the $\mathrm{NADH} / \mathrm{NAD}^{+}$ratio can also control metabolic rates; a more reduced state would be expected to slow TCA cycle flux through product inhibition and increase reduction of pyruvate to lactate, both leading to a more glycolytic state. When the data are viewed in total, it is clear that hepatocytes possess a myriad of options for the autonomous regulation of glucose synthesis and that changes in kinase activity probably represent a secondary mode of regulation that may be sufficient but are not necessary for the control of glucose output.

\section{The effect of changes in gluconeogenic gene expression on hepatic glucose output}

Just as reduced glucose output correlates with decreased energy charge, AMPK-independent reduction in the levels of mRNAs encoding the key gluconeogenic proteins PPAR $\gamma$ coactivator $1 \alpha($ PGC- $1 \alpha)$, cytosolic phosphoenolpyruvate carboxykinase (PEPCK), and the catalytic subunit of glucose-6-phosphatase (G6Pase) also correlates with a lowered energy charge state. Interestingly, by forced overexpression of the gluconeogenic master coactivator PGC- $1 \alpha$, Foretz et al. found that these changes in gene expression did not cause the reduced glucose output, but rather represented a parallel effect of depleted energy stores (12). This observation contributes to a growing body of data indicating that acute changes in gene expression are not the sole determinant of glucose output. For example, genetic manipulation of PEPCK protein levels in mouse liver has little effect on gluconeogenic flux, and in diabetic rats and in humans there is a lack of correlation between the expression of gluconeogenic genes and measures of hepatic glucose output $(18,19)$. It should be noted that several studies have appeared describing possible mechanisms by which metformin or other AMPK activators regulate gluconeogenic gene expression through $\operatorname{AMPK}(20,21)$. However, given the current data of Foretz et al. (12), as well as the frequent lack of correlation between glucose output and gluconeogenic gene expression, the contribution of these pathways to the pharmacological actions of metformin needs to be reevaluated.

\section{AMP vs. AMPK}

The lessons from the study by Foretz et al. (12) highlight the dangers inherent in accepting models based on abundant correlation, chemical inhibitors and activators, or even compelling plausibility when such data are not supported by loss-of-function experiments. Such erroneous conclusions are not novel in the AMPK field; the initial enthusiasm for AMPK as a mediator of contractionstimulated glucose uptake in muscle has not been supported by genetic experiments in vivo $(22,23)$. It is likely that elevated AMP/ATP ratio and AMPK activation represent two parallel, and at times redundant, pathways that both attempt to retain a normal energy status by exerting similar effects on metabolic pathways. Nonetheless, an alternative explanation for the AMPK-independent effects on hepatic glucose output and gluconeogenic gene expression observed by Foretz et al. (12) could be the presence of an additional energy charge-regulated kinase that could compensate for the lack of AMPK. That the effects are also LKB1 independent and that there are no other AMPK-related kinases known to be regulated by AMP makes this explanation less likely. However, novel mechanisms could allow other kinases to recognize energy charge and modulate glycolytic/gluconeogenic flux accordingly. Indeed, recent work has indicated a metformin-induced but AMPK-independent inhibition of the mammalian target of rapamycin complex 1 (mTORC1) kinase, highlighting the plurality of energy charge-responsive kinases (24).

\section{Implications for the development of therapeutic agents}

AMPK has been a target attractive to a pharmaceutical industry eager to develop new drugs for the treatment of insulin resistance and diabetes mellitus. It is important to emphasize that while Foretz et al. show that acute delivery of metformin to hepatocytes inhibits glucose output in the absence of AMPK (12), there are no data to refute the notion that pharmacologic activation of AMPK would produce the same effect without decreasing ATP levels. As noted above, genetically activated AMPK does suppress hepatic glucose output, and it is not surprising that there would exist multiple parallel pathways for eliciting critical metabolic responses. Nonetheless, these new results call for a serious reexamination of whether any other of the biological responses attributed to AMPK, which now include such critical phenomena as anticancer activity, are in fact due directly to changes in energy charge or other related kinases (25).

\section{Acknowledgments}

The authors acknowledge financial support from NIH grants R01 DK56886 (to M.J. Birnbaum) and F32 DK079572 (to R.A. Miller).

Address correspondence to: Morris J. Birnbaum, 322 Clinical Research Building, 415 Curie Blvd., Philadelphia, Pennsylvania 19104, USA. Phone: 215.898.5001; Fax: 215.573.9138; E-mail: birnbaum@mail. med.upenn.edu.

1. King H, Aubert RE, Herman WH. Global burden of diabetes, 1995-2025: prevalence, numerical estimates, and projections. Diabetes Care. 1998;21(9):1414-1431.

2. Bosi E. Metformin - the gold standard in type 2 diabetes: what does the evidence tell us? Diabetes Obes Metab. 2009;11(suppl 2):3-8.

3. Nathan DM, et al. Medical management of hyperglycemia in type 2 diabetes: a consensus algorithm for the initiation and adjustment of therapy: a consensus statement of the American Diabetes Association and the European Association for the Study of Diabetes. Diabetes Care. 2009;32(1):193-203.

4. Watanabe C. Studies in the metabolic changes induced by administration of guanidine bases. I. Influence of injected guanidine hydrochloride upon blood sugar content. J Biol Chem. 1918;33:253-265.

5. Bailey CJ, Day C. Traditional plant medicines as treatments for diabetes. Diabetes Care. 1989;12(8):553-564.

6. Goodarzi MO, Bryer-Ash M. Metformin revisited: re-evaluation of its properties and role in the pharmacopoeia of modern antidiabetic agents. Diabetes Obes Metab. 2005;7(6):654-665.

7. Ferner RE, Rawlins MD, Alberti KG. Impaired betacell responses improve when fasting blood glucose concentration is reduced in non-insulin-dependent diabetes. QJ Med. 1988;66(250):137-146.

8. Selvin E, et al. Cardiovascular outcomes in trials of oral diabetes medications: a systematic review. Arch Intern Med. 2008;168(19):2070-2080.

9. El-Mir MY, Nogueira V, Fontaine E, Averet N, Rigoulet M, Leverve X. Dimethylbiguanide inhibits cell respiration via an indirect effect targeted on the respiratory chain complex I. J Biol Chem. 2000;275(1):223-228.

10. Zhou G, et al. Role of AMP-activated protein kinase in mechanism of metformin action. J Clin Invest. 2001;108(8):1167-1174.

11. Foretz M, et al. Short-term overexpression of a constitutively active form of AMP-activated protein kinase in the liver leads to mild hypoglycemia and fatty liver. Diabetes. 2005;54(5):1331-1339. 
12. Foretz M, et al. Metformin inhibits hepatic gluconeogenesis in mice independently of the LKB1/ AMPK pathway via a decrease in hepatic energy state. J Clin Invest. 2010;120(7):2355-2369.

13. Berglund ED, et al. Hepatic energy state is regulated by glucagon receptor signaling in mice. J Clin Invest. 2009;119(8):2412-2422.

14. Tanaka T, Sue F, Morimura H. Feed-forward activation and feed-back inhibition of pyruvate kinase type L of rat liver. Biochem Biophys Res Commun. 1967; 29(3):444-449.

15. Kemp RG. Rabbit liver phosphofructokinase. Comparison of some properties with those of muscle phosphofructokinase. J Biol Chem. 1971; 246(1):245-252.

16. Van Schaftingen E, Hers HG. Inhibition of fructose1,6-bisphosphatase by fructose 2,6-biphosphate. Proc Natl Acad Sci U S A. 1981;78(5):2861-2863.

17. Taylor SI, Mukherjee C, Jungas RL. Regulation of pyruvate dehydrogenase in isolated rat liver mitochondria. Effects of octanoate, oxidationreduction state, and adenosine triphosphate to adenosine diphosphate ratio. J Biol Chem. 1975; 250(6):2028-2035.

18. Burgess SC, et al. Cytosolic phosphoenolpyruvate carboxykinase does not solely control the rate of hepatic gluconeogenesis in the intact mouse liver. Cell Metab. 2007;5(4):313-320.

19. Samuel VT, et al. Fasting hyperglycemia is not associated with increased expression of PEPCK or G6Pc in patients with type 2 diabetes. Proc Natl Acad Sci US A. 2009;106(29):12121-12126

20. Shaw RJ, et al. The kinase LKB1 mediates glucose homeostasis in liver and therapeutic effects of metformin. Science. 2005;310(5754):1642-1646.

21 . He L, et al. Metformin and insulin suppress hepatic gluconeogenesis through phosphorylation of CREB binding protein. Cell.
2009;137(4):635-646.

22. Mu J, Brozinick JT Jr, Valladares O, Bucan M, Birnbaum MJ. A role for AMP-activated protein kinase in contraction- and hypoxia-regulated glucose transport in skeletal muscle. Mol Cell. 2001;7(5):1085-1094.

23. Musi N, Hayashi T, Fujii N, Hirshman MF, Witters LA, Goodyear LJ. AMP-activated protein kinase activity and glucose uptake in rat skeletal muscle. Am J Physiol Endocrinol Metab. 2001; 280(5):E677-E684.

24. Kalender A, et al. Metformin, independent of AMPK, inhibits mTORC1 in a rag GTPase-dependent manner. Cell Metab. 2010;11(5):390-401.

25. Libby G, Donnelly LA, Donnan PT, Alessi DR, Morris AD, Evans JM. New users of metformin are at low risk of incident cancer: a cohort study among people with type 2 diabetes. Diabetes Care. 2009;32(9):1620-1625.

\title{
Radioprotection: smart games with death
}

\author{
Andrei V. Gudkov1,2 and Elena A. Komarova ${ }^{1}$
}

1Department of Cell Stress Biology, Roswell Park Cancer Institute, and ²Cleveland BioLabs Inc., Buffalo, New York, USA.

\begin{abstract}
The efficacy of cancer treatment by radiation and chemotherapeutic drugs is often limited by severe side effects that primarily affect the hematopoietic system and the epithelium of the gastrointestinal tract. Progress in understanding differences in the mechanisms involved in the responses of normal and tumor cells to genotoxic stress has led to the development of new rational approaches to selective protection of normal cells, such as suppression of apoptosis by pharmacological inhibition of p53 or activation of NF- $\kappa B$. Another promising approach presented in this issue by Johnson et al. is based on the idea of using pharmacological inhibitors of cyclin-dependent kinases (CDKs) to convert normal cells into a radioresistant state by inducing reversible cell cycle arrest at the $G_{1} / S$ transition. The evidence indicates that this approach is likely to be specific for protection of normal cells and may, therefore, have clinical potential as an adjuvant in anticancer therapies.
\end{abstract}

\section{Importance and challenges of clinical radioprotection}

While radiotherapy is a powerful anticancer treatment approach, it is associated with severe side effects that drastically limit its therapeutic capacity. Two of the most radiosensitive tissues - the hematopoietic (HP) system and the gastrointestinal (GI) tract - provide the biggest clinical challenges. The outcome of radiotherapy would be substantially improved if its therapeutic index could be increased by selective reduction of damage to the HP and GI systems, without an increase in the radioresistance of tumors. There is evidence indicating that

Conflict of interest: Andrei V. Gudkov is a shareholder in and consultant for Cleveland BioLabs Inc.

Citation for this article: J Clin Invest. 2010; 120(7):2270-2273. doi:10.1172/JCI43794. increasing the cumulative radiation dose by as little as $10 \%-20 \%$ might make a difference between incomplete and complete eradication of tumors, as shown, for example, for head and neck cancer (1). In order to allow for increased radiotherapy doses, pharmacological approaches to radioprotection of normal tissues must be developed. These approaches must be strictly selective for healthy tissues and must be safe (i.e., must not promote the development of radiation-induced secondary cancers).

Since the killing of cells by radiation is mediated by the ionization of irradiated matter, the most well-characterized principle of radioprotection involves using antioxidants, which act as scavengers of reactive oxygen species. The only drug currently approved for clinical use to protect against the toxicity of radiotherapy, amifostine, works in this way (2). However, clinical use of amifostine is complicated by its own toxicity and lack of sufficient selectivity in protection of normal versus tumor cells (2).

\section{Targeting apoptotic mechanisms for radioprotection: p53 inhibitors}

In the 1990s, it became clear that the massive cell loss that occurs in radiosensitive tissues and embryos after irradiation and leads to lethality is not due to irreversible damage of cells but rather to activation of apoptosis. This apoptosis is largely p53 dependent, and p53-deficient mice are resistant to doses of radiation that kill wild-type mice by inducing lethal HP acute radiation syndrome (3). Importantly, apoptotic mechanisms are frequently inactivated in tumors, as part of their progression toward unconstrained growth (4). These findings presented an attractive opportunity for the development of pharmacological inhibitors of p53 capable of providing reversible radioprotection by temporarily blocking p53-mediated apoptosis. The feasibility of this idea was proven by isolation of small molecule p53 inhibitors, named pifithrins, that demonstrated radioprotective efficacy in mice $(5,6)$. Since p53-dependent apoptosis is considered a major component of p53 tumor suppressor activity, concerns regarding the safety of pharmacological p53 inhibition have slowed down clinical developments based on this approach. 